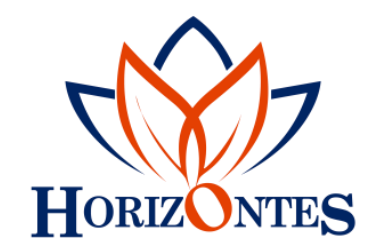

www.revistahorizontes.org

\title{
Innovaciones educativas y la tecnología educativa en la UPEL-IPC
}

\author{
Educational innovations and educational technology at UPEL-IPC \\ Inovações educacionais e tecnologia educacional na UPEL-IPC
}

ARTÍCULO DE INVESTIGACIÓN

\author{
Belkys Guzmán \\ belkys.juliana.guzman@gmail.com \\ ORCID: 0000-0002-8141-5990
}

\author{
Santiago Castro \\ castrosantiago2015@gmail.com \\ ORCID: 0000-0003-2848-0870
}

\author{
Régulo Rauseo \\ rauseor@gmail.com \\ ORCID: 0000-0002-0119-0999
}

Universidad Pedagógica Experimental Libertador. Instituto Pedagógico de Caracas, Venezuela

Recibido 01 de enero 2021 | Arbitrado y aceptado 21 de enero 2021 | Publicado en marzo 2021

\section{RESUMEN}

El objetivo de este artículo es interpretar la información recabada que permita establecer las innovaciones producidas por la incursión de la tecnología en el aprendizaje de la formación inicial, continua y permanente de los docentes en la UPEL-IPC. Se reconstruye la información a partir de los hallazgos obtenidos en una investigación de campo, dentro del paradigma interpretativo, y para ello se trianguló la información proveniente de entrevistas a profundidad, la revisión crítica de documentos y experiencias de los autores. Se encontró que la UPEL- IPC a través del DTE, desde sus inicios ha desarrollado en los docentes innovaciones educativas con base en la tecnología imperante, además muestran apertura para implementar estrategias innovadoras y medios digitales, y tomar decisiones en cuanto al diseño e implantación de las TIC, TAC, TEP para ser una educación más efectiva y eficiente.

Palabras clave: Innovaciones educativas; Tecnología Educativa; TIC; TAC; TEP

\section{ABSTRACT}

The objective of this article is to interpret the information collected that allows to establish the innovations produced by the incursion of technology in the learning of initial, continuous and permanent training of teachers at UPEL-IPC. The information is reconstructed from the findings obtained in a field investigation, within the interpretive paradigm, and for this the information from in-depth interviews, the critical review of documents and the authors' experiences was triangulated. It was found that the UPEL-IPC through the DTE, since its inception has developed educational innovations in teachers based on prevailing technology, they also show openness to implement innovative strategies and digital media, and make decisions regarding the design and implementation of ICT, TAC, TEP to be a more effective and efficient education.

Key words: Educational innovations; educative technology; TIC; TAC; TEP 


\section{RESUMO}

O objetivo deste artigo é interpretar as informações coletadas que permitem estabelecer as inovações produzidas pela incursão da tecnologia na aprendizagem da formação inicial, contínua e permanente de professores da UPELIPC. As informações são reconstruídas a partir dos achados obtidos em uma investigação de campo, dentro do paradigma interpretativo, e para isso foram trianguladas as informações das entrevistas em profundidade, da revisão crítica de documentos e das experiências dos autores. Verificou-se que o UPEL-IPC através do DTE, desde o seu início tem desenvolvido inovações educacionais em professores com base na tecnologia vigente, eles também mostram abertura para implementar estratégias inovadoras e de mídia digital, e tomar decisões quanto ao desenho e implementação de TIC, TAC, TEP para ser uma educação mais eficaz e eficiente.

Palavras-chave: Inovações educacionais; tecnologia educativa; TIC; TAC; TEP

\section{INTRODUCCIÓN}

A través de los años, los desarrollos científico-tecnológicos son los catalizadores significativos de los cambios socioculturales y de políticas que envuelven a la sociedad. El conocimiento y el saber humano emergen como un recurso capital y esencial de la educación en todos los niveles y modalidades, éstos han avanzado conforme cambian los modelos didácticos y las teorías que sustentan los procesos de enseñanza y aprendizaje. Más aún, las prácticas que llevan a cabo los docentes en el ámbito profesional que en muchos casos pueden ser innovadoras, puesto que deben mejorar la calidad educativa.

Los procesos educativos efectivos $\mathrm{y}$ eficientes requieren de una planificación y administración que contemplen innovaciones en sus estrategias, medios y recursos incluso la forma de evaluar, lo que conduce a reflexionar sobre la praxis docente, y el papel social que debe cumplir en producción y difusión del conocimiento, aunque estas innovaciones tienden a ser discontinuas como resultado de que tradicionalmente no se han evaluado e implementan desconociendo el contexto y las características de la audiencia.

En la Universidad Pedagógica Experimental Libertador -Instituto Pedagógico de Caracas (UPEL-IPC) específicamente en el Departamento de Tecnología Educativa (DTE) se han implementado diversas innovaciones que han impactado la educación venezolana de diversas maneras, pero en la actualidad hay personas que en la universidad y en esta época todavía se preguntan cuáles han sido los aportes y las bondades que han tenido estas innovaciones en la universidad y por supuesto en la educación venezolana, lo que conduce a preguntarse ¿cuáles son $\mathrm{y}$ han sido esas innovaciones y cómo se han desarrollado en el UPEL-IPC?. ¿Han impactado en el pensamiento y acción de la comunidad en la universidad? ¿Será por desconocimiento o es que no tuvieron un aprendizaje significativo, falta de promoción, difusión o es que la comunidad no ha visualizado los cambios y transformaciones? Pareciera que la comunidad requiere de más información sobre el producto alcanzado para que puedan captarlo, por lo que se pretenden difundir las innovaciones implementadas por el DTE en los últimos años.

Pareciera que algunos miembros de la comunidad consideran que, el qué aprender, el cómo y con qué enseñar ha estado allí siempre o se aprende fácilmente sin que sea enseñado al futuro docente, pero la realidad muestra que la praxis docente se construye con conocimiento y experiencia. Al respecto Suárez (2011) refiere que en la labor docente, se usan tantos elementos que provienen de la didáctica que fueron asimilados de manera formal en los estudios de pregrado y postgrado, aunado a la experiencia de aula que permite enfrentar las múltiples realidades que se presentan en el 
salón de clases. El saber diseñar, producir y usar cualquiera estrategia o medio se debe aprender y por supuesto debe ser mediado por expertos, por modelaje $u$ otras maneras que le expliquen al futuro docente su planificación, diseño, ejecución y evaluación.

Se debe señalar que innovar en la escuela ha sido a través de los años, un proceso que muchos reconocen y otros consideran como parte del trabajo diario. Una maestra que sepa dibujar y coloca en sus clases un corazón que parece latir, un ojo que parece que mira al pasar frente a él, o un barco que puede llevar a pasear al observador por el mundo solo acompañado con una historia bien contada, estos son medios que impulsan la apertura, desarrollo o cierre de la estrategia, son innovaciones que muy pocos registran puesto que "lo normal" es que una maestra sepa dibujar para enseñar ciencias y contar cuentos para la promoción de la lectura, pero no es así, eso fue planificado, desarrollado y ejecutado con el producto esperado.

Otras maestras llevan láminas y/o fotografías para motivar o guiar el aprendizaje o utilizan un pollito al que se le ve el esqueleto o un germinador o corte longitudinal para desarrollar sus clases, excelentes ayudas a diferencia de cuando se explica en forma oral sin ningún otro tipo de medio que no sea la voz. Esas ayudas son soluciones instruccionales, es decir, son innovaciones.

Hacer un cine foro, una historieta para enseñar a leer o llevar a un grupo de estudiantes a un recorrido por la ciudad o una visita a un ancianato de una comunidad para conocer de nuestras tradiciones es innovar. Como lo era en la década de los 70 y 80 aplicar un plan modular, plan Keller, enseñanza asistida por computadora, uso de simulaciones o juegos instruccionales, ahora utilización de redes sociales $\mathrm{o}$ entornos virtuales de aprendizaje.
En la planificación, diseño y desarrollo curricular se toman en cuenta las innovaciones educativas con especial atención a las Tecnologías ya que ello implica cambios y enfoques de acuerdo con las tendencias curriculares del momento.

Se debe tomar en cuenta que la innovación implica un proceso de solución de problemas y una visualización de un sistema que involucra las etapas del desarrollo tecnológico a saber; estudio de necesidades, diseño, implantación y/o simulación y evaluación. Son muy variadas, pero en este trabajo solamente se tratará de nuevas estrategias 0 medios $\mathrm{y}$ recursos didácticos que propicien una enseñanza más activa y motivadora, que desde una perspectiva sistémica, actúan como un estímulo de mejora dentro de la red compleja de interacciones.

El presente artículo tiene el objetivo interpretar la información recabada que permita las innovaciones producidas en la incursión de la tecnología en el aprendizaje de la formación inicial, continua y permanente de los docentes en el UPEL-IPC, para lo que se realiza un análisis de la integración de los medios, recursos y estrategias innovadoras en función de un aprendizaje como resultado del adecuado uso de la Tecnología Educativa.

\section{Marco teórico o revisión bibliográfica}

\section{Innovación e innovación educativa}

La innovación como propiciadora de la dinámica del desarrollo de la ciencia y la tecnología encuentra su expresión en toda la actividad humana, impregnan todos los elementos del sistema instruccional y aportan herramientas valiosas para, emprender cambios y transformaciones que repercuten en el aprendizaje (Granda, Espinoza y Mayon, 2019). La innovación es un concepto en el que las organizaciones están orientadas por el 
interés social y colectivo, es más factible la integración y cooperación, y en consecuencia es más viable la articulación de redes, o como la introducción de un nuevo producto o método, es la integración, la combinación, o síntesis del conocimiento en productos, procesos o servicios originales, relevantes y valiosos (Rodríguez, 2006).

Toda innovación logra abarcar tanto la idea de un desarrollo tecnológico como la de un cambio social, que se inicia como una imagen nueva, valiosa, creativa que se ejecuta en una organización ya sea esta educativa, empresarial, comunitaria entre otras, por parte de individuos y equipos de trabajo, porque la innovación debe ser una cultura de cualquier organización con miras a la visión y misión de esta. Un proceso interactivo para satisfacer necesidades, mediante la introducción de procesos, enfoques o metodologías, con los que se obtienen efectos exitosos e impactan en el conocimiento, los saberes y en el contexto tanto del talento humano, la organización y la sociedad.

Para Ramírez, Montoya, Valenzuela y González (2017) "la innovación educativa es un proceso que responde a las necesidades detectadas, ser eficaz y eficiente, sostenible en el tiempo y que los resultados obtenidos de su aplicación abarquen terrenos más amplios del contexto en el que fue creada" (p. 78). Estas generan transformaciones en los contextos y en los sujetos que la impulsan y sostienen.

Los docentes sustentan que, para poder innovar en las aulas, es indispensable la formación continua y la actualización permanente, además de ser creativos y en muchos casos consideran que es imprescindible tener recursos. Pero inicialmente lo que hay que tener es pensamiento sistémico para poder visualizar los problemas y cuáles son las posibles soluciones y algo primordial es no tener resistencia al cambio.
Toda innovación debe responder a las demandas sociales, atender la pertinencia, evaluación de la calidad académica en cada uno de los elementos como son planificación, administración y evaluación estratégica y por último, que emplee las tecnologías de información y comunicación. De manera que la función del docente se considera como un conjunto de decisiones que lleve a cabo el profesorado para desarrollar procesos de enseñanza y aprendizaje de calidad y promover el óptimo desarrollo de los estudiantes (García, Armenta, Lozano, Mercado, 2020).

Es indispensable que haya apertura para innovar en educación, y consideran los constantes cambios y la complejidad de las problemáticas sociales actuales, desde la visión de docentes, estudiantes y comunidad, que pueden potenciar $\mathrm{u}$ obstaculizar las innovaciones educativas en la universidad. En el marco de estas condiciones, se indaga respecto del papel de la Tecnología Educativa como herramientas para la innovación (Moreira, Abuzaid, Elisondo, Melgar, 2020).

Según la UNESCO, la innovación educativa es:
Un acto deliberado y planificado de solución de problemas, que apunta a lograr mayor calidad en los aprendizajes de los estudiantes, implica trascender el conocimiento y pasar del aprendizaje pasivo del estudiante a una concepción donde el aprendizaje es interacción y se construye entre todos. (2016, p. 3).

Muchas instituciones realizan cambios creyendo que es innovación. Por ejemplo, cambiar del fotocopiado de materiales de la clase a colocarlo en el blog del docente, es sólo 
uso de tecnología sin ser realmente innovación que impacte el aprendizaje del estudiante; el uso de la enseñanza no formal e individualizada en los años 70 como el plan Keller, utilizado en la asignatura Evaluación I, aplicación experimental realizada en el primer semestre de 1974 (Sosa, 1974) que permitía, según la innovación, que el alumno fuese a su ritmo. Fue aplicado dentro de un sistema de estudio semestral, lapso de 16 semanas máximo, lo que dejaba muy claro que el ritmo personal no se salía del espacio establecido; al finalizar el semestre, el participante debería haber cumplido con los requisitos exigidos para continuar sus estudios, era solo un cambio de formato, no una innovación dentro del sistema de estudio. (Rauseo, 2015).

Ya en los años 80, en la elaboración de Textos académicos los referentes informativos buscan usar las nuevas fuentes o tecnologías de información y comunicación. En el caso universitario, de acuerdo con las conclusiones del Seminario Multinacional "Nuevos Enfoques En El Diseño y Desarrollo de Materiales Educativos Impresos" (1983), en las conclusiones del tema 2, Problemática del libro de texto en los diversos niveles del sistema educativo latinoamericano, (pp. 42 - 48), se propone un conjunto de criterios y pautas que son aplicables a la elaboración del libro de texto. Relevante para esta investigación es lo siguiente: Las referencias bibliográficas debe estar también referidas a revistas o boletines de la especialidad como medio de ofrecer una información más actualizada que la que figura en tratados, enciclopedias, manuales y textos. (p. 47), en muchos casos menores de 5 años y de todas las esferas investigativas. En este sentido, ya se visualiza usar referencias extraídas de internet, donde se encuentra la información más actualizada, normalmente producto de investigaciones y trabajos recientes (Rauseo, 2003).
La innovación, determinada como adecuación a los elementos enmarcados por las resoluciones 12 (1983) y 1 (1996) establecidas por el gobierno de turno, acompañadas por los últimos conocimientos desarrollados sobre el movimiento humano, el humanismo y la atención a personas con discapacidades funcionales. Aparece la preocupación por la salud, el ambiente y el uso de la tecnología. Por lo que se infiere, que el contexto innovación es solo un referente administrativo de cambios y no lineamientos para el uso en investigaciones que permitan cambios en el contexto educativo. (Rauseo, 2015). Estas resoluciones buscaban la formación de los docentes con capacidad para promover innovaciones y el desarrollo además de realizar diseños pedagógicos que conduzcan a la formación del ciudadano, facilitando el proceso social, cultural, científico y tecnológico del país.

Un reto importante es estar pendiente de que entre lo pedagógico y lo tecnológico debe existir alianza y equilibrio. Ya que apoya la enseñanza tomando en cuenta el contexto, la audiencia y otros factores que afectan el proceso, facilita la mediación del aprendizaje. Lo tecnológico, además, ocupa un lugar estimable para el desarrollo del análisis y del pensamiento sistémico ya que facilita el estudio desde varias perspectivas y el desarrollo de acciones fundamentales (UNESCO, 2016). Es por ello que se debe asumir que una innovación satisfactoria en la educación está en garantizar las condiciones necesarias, los escenarios y las herramientas, con el fin de que la aplicabilidad de los avances tecnológicos.

\section{TIC, TAC TEP}

La tecnología es creada por el hombre y para solucionar problemas del humano, ha estado al lado de él durante toda la historia, pero nunca más visible que en este momento 
histórico, se abren nuevas posibilidades a la sociedad, la cultura y por ende a la educación haciendo que esta sea más efectiva y eficiente.

Muchos conocen los avances tecnológicos $\mathrm{y}$ sus aplicaciones pero sin darles nombre porque se usa para comunicarse, acceder a clases sobre todo en este momento de pandemia mundial y para consumir y producir conocimiento e información.

Las TIC (Tecnología de Información y Comunicación), TAC (Tecnología del Aprendizaje y del Conocimiento) y TEP (Tecnología para el Empoderamiento y la Participación) son muy importantes para optimizar la enseñanza por lo que se deben revisar para darle basamento a la información relacionada con la tecnología educativa.

Se comenzará por referir a Castro y Guzmán, (2020 p. 2) que señalan que autores tales como:

Lozano (2011), Montero (2014), Castañeda y Adell (2013), RM (2015), Cabero (2015) que consideran que las TIC facilitan los procesos de transmisión e intercambio de información, en todos los ámbitos de la vida incluyendo el ámbito educativo, es por ello que como necesidad educativa surge el término TAC (Tecnologías del Aprendizaje y del Conocimiento), referido al uso de las TIC como herramienta formativa, incidiendo en la metodología y en la utilización de la tecnología dentro de las planificaciones educativas. Así la apropiación de las TIC pasa de ser elementos meramente instrumentales a ser fundamentales en un sistema eficiente. Lo que hace que la escuela de respuesta a los retos a los que se enfrenta y adquiera el nuevo papel que ha de asumir, desde un paradigma inclusivo e intercultural (López, 2015).
Hay que acotar que es importante que los docentes y estudiantes se apropien de las TAC $\mathrm{y}$ de las TEP porque permiten desarrollar los conocimientos y saberes con base en el contexto potenciando así los elementos del sistema instruccional haciéndolos más efectivos y eficientes.

Las TAC son una provocación y una oportunidad para la sociedad, comunidad, nación o país y el desarrollo óptimo, creativo y crítico de los procesos de enseñanza $y$ aprendizaje dando respuesta al contexto y a la audiencia, estas impulsan a realizar cambios en todos los elementos del sistema educativo, suscitando una comunicación fluida y eficaz entre sus miembros cara a cara o a través de diferentes dispositivos, acercando y limando diferencias. Ellas permiten utilizar los medios y estrategias con propiedad $y$ en forma innovadora en aprendizaje formal e informal, además con el uso de diferentes niveles y modalidades del sistema educativo. Por ello es necesario que docentes y estudiantes se formen y tengan apertura para cambiar de roles si así es requerido. Además, se debe cambiar la forma de evaluar cómo y con qué.

Este manejo de las TAC conduce a la revisión de los currículos no solo para la formación docente, ya que esto implica, enfoques y teorías curriculares embebidas en la Teoría de Sistemas, en la integración permanente de las partes y un manejo diestro de las innovaciones tecnológicas, ya que exige un constante aprender.

Apropiarse de las TEP es muy significativo para la educación y la ciudadanía, para desarrollar el liderazgo comunitario y emprender acciones en su localidad, escuela o comunidad, para ello es imprescindible ser asertivo, líder, veraz, honesto, que acompaña la palabra con la acción, conocedor de la realidad, lo que es necesario para participar 
difundiendo y defendiendo sus derechos y deberes como ciudadano del mundo, porque las TEP no solo comunican sino que crean, consumen, producen $\mathrm{y}$ hacen tendencias transformando su entorno personal, profesional y ciudadano, es necesario que docentes y estudiantes acepten riesgos, que sean autosuficientes y tengan seguridad en su porvenir laboral. Por eso la importancia de fomentarlo en los ciudadanos de diferentes edades y de situación contextual con la intención de propiciar la autonomía, la asertividad, la creatividad y superación ante la vida, la confianza y el pensamiento sistémico y crítico, enfrentar y solucionar conflictos y tomar decisiones. Para ello la escuela debe mediar las tecnologías de emprendimiento y participación.

Saraiba (2019) considera que en estos momentos Venezuela y en general toda la humanidad requiere de ciudadanos, que ejerzan y actúen para lograr que se cubran sus necesidades, entre ellas que la realidad contextual de sus países cambie y mejoren con la meta fija de transformar la realidad, con base en los reglamentos, respetando sus deberes y derechos.

En todos los niveles, países, universidades incluso para elegir la directiva de la junta de condominio, se vota sin estar preparados para ello. La escuela debe sentar las bases que permitan construir la ciudadanía tiene como misión la formación del ciudadano entre otras cosas para que reconozca que: a) existen otras formas diferentes a las que le ha tocado vivir, b) la libertad de tránsito y de expresión son fundamentales, c) la alternancia política es sana d) es importante conocer tus deberes y derechos y d) finalmente nada ni nadie está por encima de la ley (Saraiba, 2019).

Es necesario conocer que tus derechos terminan donde comienza el de los demás, aprender a vivir en comunidad, a compartir cuando se pueda y a pensar críticamente, es decir, formar la conciencia ciudadana. Entonces se deben desarrollar estrategias instruccionales en las cuales se utilicen técnicas interactivas en las cuales se les planteen problemas a los estudiantes y estos participen y contribuyan efectivamente a su solución. Es importante que cada actividad gire en torno a lo que plantea Saraiba (2019):

1. Preguntarles qué piensan sobre la situación ¿qué conocen?, ¿qué saben?, ¿qué manejan? como punto de partida desde el cual puedan aclararse dudas, completarse vacíos e intercambiar ideas.

2. Recordar cómo deberían ser las cosas no perder de vista los referentes de la sociedad lo que debería ser, recordando que el hecho de que algunas personas no cumplan con su deber no significa que ese deber no exista.

3. Evitar reproducir tus ideas y posturas: es muy importante preguntar, repreguntar, parafrasear para tratar de favorecer que estos desarrollen su punto de vista, coincidentes y sino, desarrollar la tolerancia ante las diferencias.

4. Evitar ofender $y$ que ofendan o humillen: se debe evitar el uso de calificativos o insultos. Se puede decir que no estoy de acuerdo e inclusive molesto, debe permitir que forme su propio criterio.

5. Reconocer mis límites: Es necesario enseñar: a) reconocer errores, b) reconocer que no se maneja toda la información y que puede buscarse c) tener apertura ante el error o desconocimiento.

En fin, las TEP deben ser usadas para fomentar la participación de los "ciudadanos" en diferentes temas de índole político o social generando de esta forma una especie de 
Empoderamiento y concientización de su posición en la sociedad que se traduce en expresiones de reclamos de sus derechos humanos y/o acción pública.

Es necesario tener una capacidad reflexiva crítica para ello hay que subsanar la necesidad de formación, con el fin de adaptarse a los avances vertiginosos que lo abruman y le exigen modificar y asumir con apertura, para ello debe apropiarse y manejar con propiedad las TIC, TAC y TEP, en la búsqueda de optimizar su desempeño en el trabajo, de manera eficaz y eficiente. Estas tecnologías incluyen el uso de representaciones apropiadas, conocer y aplicar los modelos que son esenciales en el aprendizaje, la utilización de juegos y simulaciones, tanto de la cotidianidad del estudiante como de las acciones que se quieren desarrollar, en este sentido es fundamental saber cuándo segmentar y cuando trabajarlo de manera global.

La enseñanza para la reflexión y el pensamiento crítico requiere de un docente que genere su propio entorno, ya que debe entender que la enseñanza es un proceso de construcción cooperativa que se genera en la clase con los sujetos implicados. (Rauseo, 2004). En esto influye actualmente en alto grado el manejo de TIC, TAC y TEP para el desenvolvimiento del docente en su aprendizaje inicial, continuo y permanente.

Con respecto a la relación entre la formación en TIC y su uso como estrategias para la praxis profesional, se destaca entre los encuestados que una adecuada y correcta formación, puede proporcionar múltiples ventajas a los docentes. Además, las actividades en el aula se podrían enriquecer con actividades más motivadoras y lúdicas. Es por ello que se debe fortalecer la actualización y formación de los docentes, y no solo, limitar la tenencia de experticia tecnológica y equipos en las instituciones escolares (Machado y Rojas, 2018).

La llegada e impacto de las Tecnologías en el aula redefinen el concepto tradicional del currículo, aula, relaciones de enseñanza y aprendizaje e innovación educativa, dejando atrás la secuencia instruccional lineal para pasar a la red no jerárquica, el orden de la clase basada en diferentes tipos de materiales y formatos: transparencias, diapositivas, láminas, libros impresos, videos entre muchos otros para abrirse a la gama de multimedios e hipermedios que abren el panorama a diversas formas de abordar el conocimiento tomando en cuenta las inteligencias múltiples, los estilos de enseñanza y de aprendizaje, entre otros.

Cambian los roles de los actores del sistema por acción de la conectividad y los dispositivos móviles de conexión inalámbrica, lo que permiten que el aprendizaje suceda en cualquier lugar y momento (casa, trabajo, oficina), además obliga a repensar la formación del docente con una verdadera apropiación personal y profesional de las TIC, es decir tener la necesidad de TIC igual como necesitas el celular o el internet. Mientras más se use como algo natural a diario más cambia la forma de internalizarla y usarla en todos los ámbitos.

La cultura y uso de las TIC es diferente en los ciudadanos y está cargada de factores emocionales, creencias y mitos, con respecto a la función docente y el uso de las tecnologías en todos los ámbitos de la vida, se provocan cambios y transformaciones sociales, culturales y económicas permitiendo su uso en los procesos de enseñanza y aprendizaje ya sea presencial, mixta o a distancia, en forma uni o bidireccionalmente, y propiciando el intercambio de roles y mensajes, es decir consumen, producen y distribuyen información ( Casado, Castro y Guzmán, 2007). 
Los niveles de apropiación de las TIC que tienen los docentes y estudiantes, son diferentes llegándose el caso de que los estudiantes se consideren expertos mientras el docente se inicia en el tema. Pero hay docentes que se especializan en el uso de aulas virtuales pero que no saben hacer una presentación o utilizar las herramientas comunicativas, e incluso le restan importancia al consumo y producción de contenidos publicados en las redes sociales.

Un ejemplo de lo anterior son aquellos docentes y estudiantes que dicen que usan las TIC con propiedad, pero cuando se ven los productos tales como uso de Word, búsqueda de información, o cualquier otra tarea "rutinaria" se observa un desempeño deficiente, incompleto sin ortografía correcta, márgenes inadecuados o presentaciones ineficaces y poco motivantes, entre otros errores, es decir que no demuestran esas competencias, razón por la cual se debe hacer énfasis en la formación continua y permanente del docente en estos aspectos.

Con la mirada puesta en el futuro es necesario que los docentes desarrollen competencias en el diseño, aplicación y evaluación de medios, estrategias a los diferentes canales de percepción de los estudiantes y escenarios educativos, $\mathrm{y}$ comunitario apoyados en las TIC, TAC, TEP haciéndolos más significativos, pertinentes y adecuados a los diferentes contextos.

El uso de las TIC, TAC, TEP, permiten construir el conocimiento y los saberes, entre otras cosas por medio de la búsqueda, procesamiento, almacenamiento y recuperación de la información; propician el trabajo colaborativo, al desarrollar competencias operativas, de pensamiento sistémico y crítico y producir estrategias innovadoras, medios $\mathrm{y}$ recursos en diferentes formatos. Se debe actualizar y capacitar a los docentes en el desarrollo de proyectos productivos, inclusive el emprendimiento, empoderamiento $\mathrm{y}$ productividad, serán habilidades que le permitirán mejorar su rendimiento académico, mejorando su calidad de vida, y la de su entorno (Espinosa, Rodríguez y Olvera, 2017; CamposRiusa y Sebastiani Obradorb, 2019).

La falta de información real, creíble, veraz y confiable por medio de interlocutores de reconocida honestidad informativa y la imposibilidad de tener y/o verificar la misma por otras vías, se subsana validándola por medio de la acción directa de la radio, TV, cine, redes sociales en fin usando las TIC, TAC, TEP con todas sus propiedades, características y potencialidades.

Estas tecnologías son un gran adelanto en la educación con elementos interactivos $\mathrm{y}$ didácticos para el desarrollo de actitudes y aptitudes en el entorno escolar, hacer más eficiente el modelo educativo forjando un nuevo modelo de escuela que responda a las necesidades del país y de los ciudadanos.

Es importante que a los docentes se les forme en el diseño de propuestas concretas de intervención en los diferentes niveles de planificación educativa, que vincule la teoría y la práctica, profundice en la búsqueda de los métodos y estilos de enseñanza y aprendizaje más apropiados en función de los elementos del sistema instruccional, propicie la empatía, la colaboración, afinidad, interacción social, comunicación creativa, la cultura digital, la creación de contenido y la interactividad.

Como se ha dicho son las TIC, TAC y TEP parte de los impulsores de este milenio que permite difundir la información (a través de audios, videos, sonidos, historietas, cuentos entre otros muchos archivos) para usarla en investigación, docencia, extensión, en actualización, capacitación y profundización, hacer sus cuestionarios y encuestas para 
usarlas en sus investigaciones, oír y/o compartir su música favorita o hacer una infografía para sintetizar la información. Además, realizar sesiones de intercambio de información de asesorías personalizadas, guías y mucho más para acompañar a los docentes y estudiantes en la virtualidad, o la preparación y ejecución de reuniones o clases con el uso de dispositivos, así como también el desarrollo de experiencias en modalidades como E-learning, M-learning y B-learning.

Para finalizar este aparte se muestra el gráfico 1 con los usos más relevantes de las tecnologías en la educación, aunque en estos momentos se perfilan a mucho más.

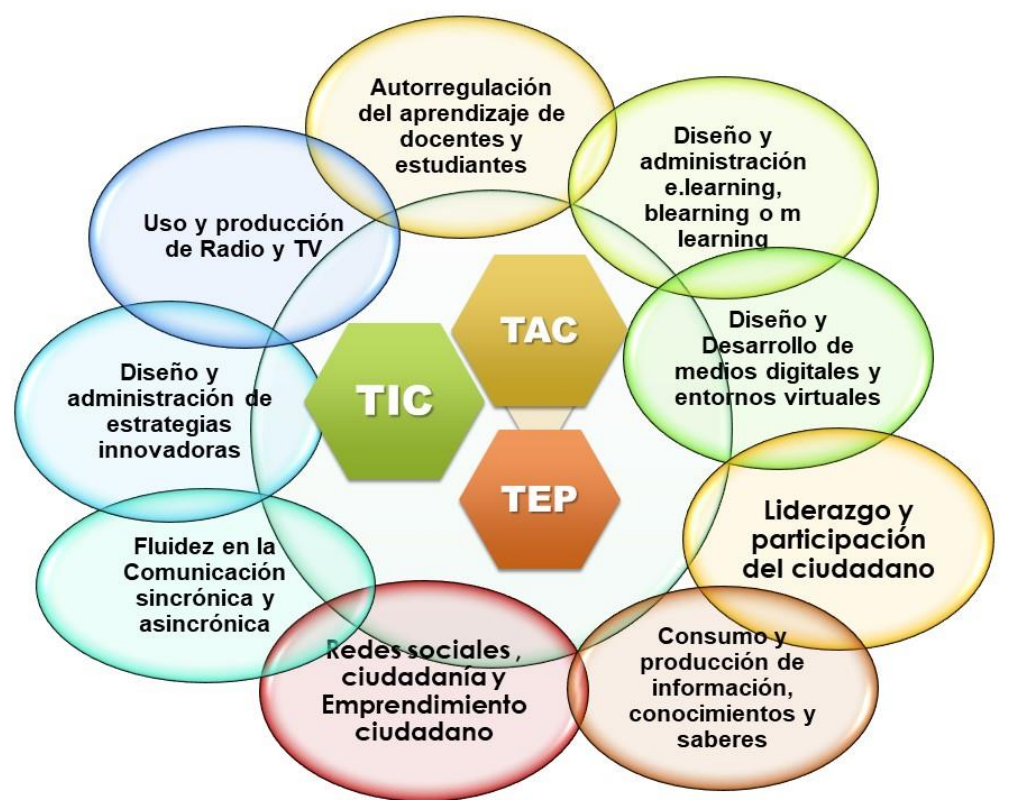

Gráfico 1. Las TIC TAC TEP en Educación.

Es importante destacar que en este artículo se explicita el conocimiento implícito relacionado con algunos de estos aspectos.

\section{METODOLOGÍA}

De acuerdo con los propósitos del estudio esta es una investigación de campo, enmarcada en el paradigma interpretativo, que permitió recopilar insumos de los informantes clave, el conocimiento del contexto y de la situación estudiada que se aborda en la problemática y, al mismo tiempo, se considera la información desde diversas perspectivas. Se realiza una triangulación tomando en cuenta el análisis de la percepción de los actores, un análisis de contenido de la documentación revisada aunada a la experiencia de los investigadores.

Este trabajo se realizó en varias fases:

a) Análisis de la documentación referencial: En esta fase se realizó una revisión crítica del estado del arte del conocimiento sobre las diferentes 
posiciones teóricas en relación con los temas: innovación, TIC, TAC y TEP.

b) Experiencia de docentes de la UPEL- IPC autores de este trabajo que estudiaron y se desempeñaron varios cargos académicos y administrativos tanto en el IPC como en educación media y

c) Entrevista en profundidad aplicada a profesores y estudiantes del DTE en el IPC.

\section{RESULTADOS Y DISCUSIÓN}

A continuación, se presentan los hallazgos de la investigación, después de realizar un análisis e interpretación de estos a partir del proceso de reducción y comparación constante de la información facilitada por los informantes clave en las entrevistas. Los resultados muestran una imagen clara y representativa de la realidad del momento. Se debe señalar que al tener como norte que el DTE y la tecnología impartida en él, es el sistema abierto que ocupa esta investigación y reconoce que fluye la información. La presentación de los hallazgos obtenidos luego de realizar la triangulación se explica a continuación.

\section{Innovación educativa del IPC y tecnología educativa}

Una de las categorías encontradas es la innovación y la tecnología educativa que según los informantes son simbióticas y avanzan en la misma medida "sabemos que si se usa la tecnología se hace innovación". "Si se usan con calidad los aparatos electrónicos como el video beam se hace innovación", pero en ambos casos no se muestra la relación entre estos términos en directo pues la tecnología por sí sola no implica innovación para ello se requiere optimar el uso de estrategias y medios.
A pesar de toda la incursión de la tecnología en todos los ámbitos de la vida, especialmente en el educativo, lo cierto es el hecho de que la mayoría de las personas tienen una idea poco clara de lo que es "Tecnología", y de sus posibilidades de aplicación en el ámbito educativo, lo que ha generado conflictos, en cuanto al enfoque tecnológico, en el campo educativo.

Consideran los informantes que la Tecnología Educativa para el colectivo de la UPEL-IPC es un concepto desconocido, la información varía entre la Tecnología no es más que aparatología inicialmente aparatos de enseñanzas y en la actualidad el uso de computadoras, aparatos eléctricos $\mathrm{y}$ electrónicos, entre otros, y esto no es diferente, en el campo educativo en el cual muchos "expertos" y "autoridades" en educación que creen que la única aplicación que tiene, es el uso de medios y recursos audiovisuales, una concepción de Tecnología Educativa de los años ochenta, mientras que la Tecnología Educativa se identifica inicialmente con los medios (Colom 1986, p. 22), luego se plantea como diseño de estrategias, uso de medios y control del sistema de comunicación (Bartolomé, 1988) y luego bajo la egida de la Teoría General de Sistemas que contribuye con herramientas y conceptos de organización a la Tecnología Educativa" (Chadwick, 1978, p. 16).

Hoy día se conoce que la Tecnología Educativa es una disciplina integradora, viva, holística y significativa de la Educación y es un campo que implica un proceso creativo interdisciplinario y sistémico que utiliza ciencia, tecnología y experiencia para producir soluciones a problemas específicos y satisfacer necesidades, optimizar la operatividad del sistema educativo (Cabero, 1999 y Szczurek, 1990). Es necesario que todo docente lo entienda y lo aplique con propiedad. 
Según los informantes clave, el saber tecnología le abrió un abanico de opciones que les permitió tener acceso a diferentes campos "como maestra enseñé a mis maestras y aplique con mis estudiantes con esos medios efectivos, ahora los enseño y aplico en mi carrera" (Morales en entrevista personal, agosto, 2020).

\section{Sistema instruccional y pensamiento sistémico}

La siguiente categoría se visualiza en la complejidad de la teoría de sistemas asumiendo que el aprendizaje ocurre en un laberinto que varía según el contexto y los actores de un sistema instruccional, esta categoría es pensamiento sistémico y el sistema instruccional. Se comenzará por señalar que la experiencia de los autores conduce a referir que una pregunta reiterativa cuando un estudiante de la maestría hace su presentación pública es ¿qué puedes decirnos acerca de qué aprendiste de la maestría en educación mención Tecnología y Desarrollo de la Instrucción, cómo ha impactado tu vida?, y la respuesta generalmente no varía "me cambió la vida y la forma de ver las cosas como un todo", y "me permite imaginar el sistema estudiando el mismo como un todo y visualizando las posibles soluciones". "Parecerá mentira, pero ahora veo las cosas de otra manera visualizando el todo y analizando cada parte" es decir, ahora tienen un pensamiento sistémico que es "una forma de pensar, que enfatiza el sistema total en vez de sus componentes, optimizando su eficacia (Raffino, 2019).

Estos estudiantes internalizan la ideología de los creadores de la maestría y ahora perciben la realidad como una totalidad para su interpretación, análisis, comprensión y por consiguiente tomar decisiones y accionar, esto permite la comprensión, simular, hacer pruebas piloto y el manejo de diferentes sistemas como existen en empresas, negocios, escuelas, sección o área de trabajo.

Es la actitud del ser humano, que se basa en la percepción del mundo real en términos de totalidades para su análisis y comprensión. Es así como se debe ver el sistema instruccional y cada uno de sus subsistemas que según Szczurek, (1989) son 6 a saber: elementos directrices (competencias, objetivos, propósitos entre otros), características biopsicosociales de docentes y estudiantes, estrategias, medios y otros recursos y evaluación, subsistemas interrelacionados entre sí en sinergia y que al desarrollar uno de ellos recursivamente afecta a los otros subsistemas y al sistema mayor para poder tomar en cuenta todos los factores $\mathrm{y}$ visualizar las innovaciones en función de cada uno de los elementos.

Como se sabe, así como el contexto y la cultura han cambiado por el efecto de los avances en la ciencia y la tecnología a través del tiempo, así también cambian los fines y propósitos de la educación y por ende esto ha modificado y afectado a todos los elementos del sistema.

Los informantes clave consideran que es difícil, pero en muchos casos se cambian los roles entre docentes y estudiantes, el docente que es un mediador de aprendizajes debe diseñar ambientes y experiencias de aprendizaje para los estudiantes, fomentar la interacción de los mismos, el autoestudio y la motivación, juega un papel muy importante en el diseño de medios y recursos que deben ser adaptados a las características de la audiencia, ya que el estudiante es un productor $\mathrm{y}$ consumidor de conocimientos y saberes, puede modificar el ambiente de aprendizaje para su desarrollo y mostrar al docente otras maneras de acceder y mediar el conocimiento. 


\section{Estrategias, medios y recursos}

La siguiente categoría se visualiza con dos subcategorías: estrategias y medios y recursos.

\section{Medios y Recursos}

En este trabajo se asume que los medios y otros recursos son el "con qué" de los sistemas de enseñanza y aprendizaje, son cualquier dispositivo o equipo que tiene la información y se usa para transmitirla entre las personas, tienen las funciones de: proporcionar información, desarrollar habilidades, motivar, despertar y mantener el interés, y propician la observación, expresión y creación, son mediadores entre la realidad y los estudiantes (Szczurek, 1989 y Marqués, 1999; 2011). Ahora bien, según los informantes clave los medios y recursos son

...un gran aporte, porque te da una serie de productos que pueden estar medianamente bien, como en toda investigación empieza con una revisión de documentos, se tiene una idea hacia donde apunta el aprendizaje, por lo que ya tienes una guía, te da basamento, te paseas por lo que puedes hacer o no puedes hacer, te indican el camino a seguir y las metas que se esperan alcanzar (Morales, M en entrevista personal agosto 15, 2020).

En la UPEL-IPC se le media al futuro docente la adopción, adaptación o producción del o los medios requeridos según el canal sensorial, control que tiene el docente del medio, su utilidad, para grandes o pequeños grupos de estudiantes, tipo de soporte que requieren. Se han desarrollado muchos medios que han sido clasificados de diferentes maneras, en este trabajo solamente se hará referencia a
Guzmán (2013, 2018); Castro y Guzmán (2020) como medios que requieren del uso de soportes computarizados tales como:

- Edu-blogs. Blogs bitácora que pueden albergar cantidades de contenidos interrelacionados mediante videos, imágenes u otros archivos, como por ejemplo los que menciona Cuberos (2020) en su reseña de Blog donde se proponen dieciocho herramientas para generar cambios en la educación con el uso de las TIC, TAC y TEP para cambiar la clase (https://www.capacitaciondocentecontic.ne t/singlepost/2019/03/31/TIC-TAC-TEPherramientaspara-cambiar-tu-clase), Blog que hace un pequeño recorrido sobre las TIC, TAC y TEP y concluye interrelacionando las tres definiciones con una visión particular del tema. https://www.conasa.es/blog/tic-tac-teptecnologias-para-la-vida/.

- Sitios web. Conjunto de páginas web, imágenes, vídeos u otros archivos como por ejemplo los que menciona Dilone (2020) en su reseña como por ejemplo los niños resuelven rompecabezas arrastrando $\mathrm{y}$ soltando para crear una historia que resuelva un problema específico. https://blockly.games/, Mundo Primaria que surge como una fuente de juegos y otros recursos didácticos gratuitos de gran calidad para niños desde 3 años de edad. https://www.mundoprimaria.com/

- Radio educativa. Enfocada a la población excluida y que buscaba soluciones a problemas sociales. En la reseña presentada por Tovar (2021) se muestran varias radios educativas vía internet entre ellas se encuentra la Radio UPEL. Circuito Nacional digital, cuya dirección es http://campus.upel.digital/index.php/radio- 
upel/ la cual se concibe como un medio de comunicación radial, para informar, entretener, educar a través de cursos, talleres, diplomados y micros en video, es un aliado estratégico de la Universidad con las comunidades que atiende, además tiene un canal de YouTube que se puede visitar en https://www.youtube.com/channel/UCrIz5 tapG6NQHLQvLVCbMvw y un grupo abierto de Facebook.

- E-book libro-es. Un texto digitalizado que tiene como soporte un archivo electrónico en vez de papel, como por ejemplo por ejemplo Promoción de la salud sexual y reproductiva, manual para facilitadores. (González y Guzmán, 2010). Los Peligros de Internet: Guía para conocerlos y enfrentarlos (Dilone y Delgado, 2020)

- Podcast. Consiste en la distribución de archivos multimedia o hipermedia (audio o vídeo que suelen ser de corta o larga duración, y los contenidos en formato $\mathrm{CD}$ ROM. DVD o memorias USB, pueden ser usados desarrollar temas de diferentes cursos, talleres o diplomados con suficiente información y enlaces para su ampliación.

- Impartir, investigar y evaluar contenidos. Con el uso de Webquest

- Las Redes Sociales. Conforman una cultura tecnológica en las sociedades, su aporte en todas las dimensiones del acontecer humano y su estructura permite a los usuarios acceder a la información, incorporar datos y tener un amplio repertorio referencial para saber que ocurre, tomar decisiones, una mejor observación en la comunicación e interacción, además conformar grupos de: trabajo, intereses, hobbies, entretenimiento, participación y emprendimiento (López, 2016; López y Guzmán, 2019; 2020).
Por otra parte, los cambios curriculares deberán convertirse en procesos ágiles, viables, permeables a las necesidades y expectativas. La educación es una de las actividades humanas más resistentes al cambio, necesita modelos que se renueven en la medida que cambian los elementos fundamentales sociales, todo con la finalidad de hacer exitosa la institución socialmente. Resistencia al cambio que puede disminuirse con formación, capacitación y una actitud positiva hacia los procesos de cambios creativos, comprometidos con la mejora del proceso formativo y sus resultados. Para ello debe involucrarse a los actores del sistema.

La innovación educativa bastante compleja, ya que dentro del mundo educativo se confunde de múltiples manera, éstas van desde el manejo de tecnologías comunicacionales de avanzada, hasta enfoques de enseñanza y aprendizaje, que sólo provocan confusión con lo que realmente sería una actitud positiva hacia los procesos de cambio por el uso de las tecnologías o métodos pedagógicos considerados novedosos, más que modelajes de enseñanza que con el tiempo se vuelven intrascendentes o se plantan como si solo de esa manera se pueden lograr dichos aprendizajes.

Respecto de las relaciones entre innovación y TIC aparecen diferentes posturas entre los participantes. Para algunos son indispensables para generar procesos innovadores en la universidad, y otros dudan de su efectividad si no está acompañado por objetivos orientados al cambio. Es decir, su incorporación no garantiza innovaciones, los cambios dependen de las perspectivas de los actores y los objetivos educativos que se plantean (Moreira, Abuzaid, Elisondo, Melgar, 2020). 


\section{Estrategias}

La otra subcategoría es la estrategia instruccional como componente del sistema de enseñanza y de aprendizaje, es un subsistema que se relaciona con los demás elementos del sistema en forma sinérgica que influyen y es influida por las decisiones y modificaciones que se realicen en cualquiera de los otros subsistemas.

Las estrategias son definidas por Montoya (1997, s/p) como: "el conjunto de técnicas, procedimientos, actividades y recursos propuestos en función del logro de los objetivos". Salcedo, (1974, en Montoya, 2013) las define como "un conjunto de acciones deliberadas y arreglos organizacionales para llevar a cabo la situación enseñanza aprendizaje". Mientras que Szczurek, (1989) define estrategias como el conjunto de acciones metodológicas que se planifican para una situación instruccional con el fin de alcanzar los objetivos preestablecidos.

Luego de contrastar e interpretar la información presentada por los informantes clave en cuanto a las estrategias se obtiene que ellos consideran que: la atención individualizada o grupal depende de las necesidades de cada participante y por ende contribuye con el desarrollo eficaz del curso. En efecto, se considera que la atención individualizada permite que cada participante consulte sobre sus dudas en el momento y las veces que necesite, esto fortalece el trabajo que realiza cada estudiante porque le ayuda a concentrarse en su aprendizaje. "Pero a su vez le permite al docente atender las necesidades particulares de cada estudiante, monitorearlo y darle el acompañamiento que requiere. En el momento que considere oportuno y las veces que considere necesario". (Y, Chirinos entrevista personal febrero 5,2018 ).
"Si el curso es de la modalidad mixta, debíamos asistir todos con nuestras diferencias individuales e intereses distintos, allí se promueve la intrasubjetividad ya que habían cosas que tu creías que no podías hacer pero con una buena guía y además recibir las críticas constructivas de tus compañeros, ese compartir de experiencias y saberes era nutritivo, desde lo intrasubjetivo más lo intersubjetivo se generaba una afinación de los diferentes productos y temas ambientales tan distintos" (H González en entrevista personal febrero 15,2018 ).

Cabe señalar que las estrategias que se planifiquen y administren en cualquier curso taller deben ser cónsonas con las características biosicosociales de los subsistemas docente y estudiantes, el nivel y la modalidad. Se requiere que en toda clase se cree un clima afectivo y altamente flexible que se preste para el intercambio tanto a distancia, mixto como presencial de los participantes y el docente, así como de los participantes y los medios, ajustadas al contexto y a las variantes de los autores del sistema.

En relación con la interacción entre los elementos del sistema se propicia con la ayuda de un acompañamiento y monitoreo efectivo por parte del docente, un aprendizaje que propicia, el crecimiento personal, la reflexión y la transferencia a diferentes contextos.

Las estrategias empleadas en las clases presenciales o a distancia, sin importar si son individuales o grupales deben generar un ambiente de respeto, cordialidad, un buen clima afectivo propicio para que se dé el aprendizaje, y respeto entre los pares y con el docente, guardando las diferencias individuales y el tiempo de ejecución de cada uno, además un clima de libertad y democracia para tomar decisiones, cónsonas con el perfil del egresado, incentivan la creatividad y la autorregulación del aprendizaje.(Guzmán, 2013). 
La interacción entre los elementos del sistema es primordial para un desarrollo adecuado, un clima agradable, un trabajo efectivo, así como también un ambiente propicio de creatividad donde todos son respetuosos y tratados con justicia, equidad y democracia, permite mayor productividad, originalidad y calidad. Esa interacción entre los actores del sistema permite la construcción de códigos con características específicas de uno y otros que les amplia los canales de comunicación y por ende propician una mejor interacción entre ellos. Cuando los estudiantes con un trabajo común desarrollan un clima afectivo con el docente y sus compañeros, se compenetran, ayudan, aunque permanezca la individualidad, ven al grupo como una unidad, esto se logra con motivación, interacción y transferencia del aprendizaje a diferentes contextos. A continuación, se detallarán algunas estrategias innovadoras, conceptos y ejemplos.

- Aprendizaje invertido. Es un método, referido a invertir la forma en que se enseña y aprende, en donde el uso de herramientas multimedia, el vídeo o el podcast, o sencillamente internet, es fundamental para que las actividades que se deben desarrollar en la casa se trabajen en el aula, apoyados del trabajo colaborativo, aprendizaje basado en problemas y proyectos y viceversa. (Martínez et. al., 2014). Esto mejora la experiencia en el aula al impartir la instrucción directa fuera del tiempo de clase. Por ejemplo, realizar una investigación individual que luego será discutida en una clase, un laboratorio no estructurado en el cual el estudiante debe realizar un proyecto que será la base para la discusión grupal en la clase, el aprendizaje de destrezas básicas en disciplinas deportivas y luego su demostración, discusión y práctica colectiva, entre otros.

- Aprendizaje Cruzado. Se lleva a cabo en entornos informales, recolectando experiencias cotidianas, tales como museos y clubes extracurriculares, vinculando el contenido educativo con cuestiones que sean atractivas a los estudiantes y que se puede profundizar agregando preguntas $\mathrm{y}$ conocimientos del aula, despertando un mayor interés y motivación para aprender (Innovating Pedagogy, 2016). Aprovechando las experiencias en múltiples entornos, con ellas los estudiantes pueden registrar, vincular, recordar y compartir sus diversos eventos de aprendizaje como por ejemplo recorridos religiosos, visitas virtuales o no a museos, ciudades, parques y jardines o cortes transversales de un terrero entre otros, para que los estudiantes puedan experimentar el aprendizaje lo más cercano a la experiencia.

- Stortelling. Arte de contar historias combinando la narrativa tradicional, la tecnología y las emociones para contar historias. Aplicar esta técnica en las aulas aumenta la motivación, la creatividad y entrena en las competencias digitales de los estudiantes, se logra, también, un ambiente más relajado y participativo en el aula. La creación de una historia con la que los aprendices conectan, incentiva su motivación, promueve su curiosidad y crean un vínculo más cercano con el docente (Educación 3.0, 2019). Algunos ejemplos de esto son las visitas de cuenta cuentos, o ancianos de la localidad para que cuenten como era la vida del lugar. Programas en el cual un docente o experto echa el cuento de la vida de un investigador caso Marie Curie, por ejemplo. 
- Aprendizaje Basado en Problemas. consiste en plantear problemas relacionados con diversas áreas de estudio para que él o los estudiantes puedan resolverlos en forma individual o grupal, de manera autónoma permite identificar los conocimientos y habilidades que hay que aprender así la enseñanza se vuelve mucho más activa y participativa, enseña a ser autónomos, independientes $\mathrm{y}$ a tener pensamiento crítico entre otros muchos beneficios. Por ejemplo, los laboratorios no estructurados donde debe investigarse, implementarse el aprendizaje colaborativo con intercambio de opiniones, la coordinación y la aceptación de críticas y valoraciones de grupo.

- Aprendizaje Basado en Proyectos (APB). Se plantea una pregunta guía que servirá para planificar y estructurar el trabajo, este se puede dividir en la selección del tema, la formación de equipos, la definición del reto final, la planificación, la investigación, el análisis, la elaboración del producto, la presentación, la respuesta colectiva $\mathrm{y}$, por último, la evaluación como por ejemplo un ejercicio de investigación. Desarrollo de proyectos de aula o lúdicos (EDUforics, 2017 y Aula planeta, 2015).

- Aprendizaje Basado en Juegos. Es una técnica de aprendizaje que traslada la mecánica de los juegos al ámbito educativoprofesional con el fin de conseguir mejores resultados, puede ser usado para desarrollar algunas competencias o mejorar otras. En muchas asignaturas se trabaja con juegos estructura, caso de juegos basados en la estructura del dominó o el monopolio para tratar diferentes temas, juegos de memoria. En otros casos juegos como el solitario que se creó con la idea de que las personas se acostumbraran al mouse y quedó como una parte divertida del uso del computador.
Utilización de juegos tradicionales para desarrollar algunas habilidades y destrezas.

Existe una gran variedad de estrategias interactivas que permiten la interacción y participación activa de los estudiantes, en este momento donde la pandemia sobrepasó los límites y ha obligado a trabajar en educación a distancia, es entonces, cuando el docente tiene que esforzarse en conocer y para desarrollar diferentes estrategias para que el estudiante tenga la posibilidad de alcanzar el aprendizaje. Lo que implica un nuevo rol del docente y del estudiante, haciendo énfasis en su relación y probable impacto en la gestión innovadora de los recursos tecnológicos y la educación virtual. (Guzmán, 2013 y Gil Mateos, 2020).

El uso de las TIC: manejo de los equipos y sus programas tanto en lo simple como en lo complejo, con la intención de hacer comprensible y emotivo el aprendizaje, como elemento esencial para hacer más eficientes los procesos que se dan. En ese sentido los entornos virtuales colaborativos son fundamentales y una necesidad en los procesos de comunicación, enseñanza y aprendizaje (Stojanovic, 2009).

\section{Consideraciones finales}

El departamento de tecnología educativa en el transcurso de su existencia en el IPC y desde su incorporación a la UPEL, ha utilizado, modelado y mediado las tecnologías vigentes en cada época de su historia, aplica y desarrolla en los individuos nuevas teorías de enseñanza y aprendizaje, toma en cuenta las características holísticas de docentes y estudiantes para desarrollarlas y adaptarla en clase. Además, muestra que es imprescindible entender la enseñanza como un sistema para facilitarlo; como un medio más que como un fin en sí mismo. 
Se debe considerar en el diseño y desarrollo del currículo de formación inicial, continua y permanente con el uso de las TIC, TAC y TEP como elementos fundamentales de la innovación educativa contextualizada con los avances del desarrollo social producto de la ciencia y la tecnología. Para ello es necesario sistematizar algunas competencias para desempeñar las funciones de docencia, investigación y extensión, desarrollar otras que a nivel mundial ya se están poniendo en práctica. Para ello se debe diseñar, ejecutar y evaluar una serie de cursos, talleres, actividades de extensión y diplomados que permitan capacitar y/o actualizar el talento humano y que multiplicará de forma efectiva el aprendizaje obtenido.

\section{REFERENCIAS}

Aula planeta (2015) El aprendizaje basado en proyectos Recuperado https://www.aulaplaneta.com/2015/02/0 4/recursos-tic/como-aplicar-elaprendizaje-basado-en-proyectos-en-diezpasos/

Bartolomé, A. (1988). Concepción de la Tecnología Educativa a finales de los 80 . Recuperado http// www.doe.d5.es/te/Any88/bartolome_tit

Cabero, J. (1999). Tecnología educativa, Editorial Síntesis, Madrid

Campos-Riusa, J., y Sebastiani Obradorb, E., (2019). Metodologías innovadoras y Tecnologías del Aprendizaje y el Conocimiento (TAC) en la materia Didáctica de la Educación Física en el Grado de Ciencias de la Actividad Física y el Deporte de la FPCEE Blanquerna - Universidad Ramon Llull congreso In-Red 2019 UPV, Recuperado

Doi: http://dx.doi.org/10.4995/INRED2019.201 9.10373

Casado, D., Castro, S. y Guzmán, B. (2007). Las TIC En Los Procesos De Enseñanza Y Aprendizaje. [Revista en línea] Revista Laurus, Año 13, Número 23, Recuperado de: http://www.redalyc.org/pdf/761/7610231 1.pdf

Castro, S., y Guzmán, B., (2020) Los medios instruccionales, su desarrollo e importancia en la educación del siglo XXI Delectus, 4(1), 1-16. Recuperado de https://doi.org/10.36996/delectus.v4i1.35

Chadwick, C. B. (1978). Tecnología Educacional para el docente. Buenos Aires: Paidós.

Colom Cañellas, A.J. (1986). Pensamiento tecnológico y teoría de la Educación. En J.L. Castillejo y Ot., Tecnología y Educación. Barcelona: Ceac. (13-30).

Cuberos G., (Septiembre-Diciembre, 2020) Reseña digital sobre las TIC, TAP y TEP Revista Investigación 101(44) 307-309 Recuperado de https://es.calameo.com/read/006246799e 69ff007e73e 3017-309

Dilone. D, (2020 mayo-agosto) Reseña Programación en niños en edad prescolar Revista de Investigación 100(44) 246-248 Recuperado

https://issuu.com/daliadiezdetancredi/doc s/finalisima_rev._inv._100.vol_44_a_o_2020_ _mayo_ago

Dilone D, y Delgado. N., (2020) Producción de Ebook Interactivo sobre los peligros de Internet: guía para conocerlos y enfrentarlos. Revista de Investigación 100(44) 246-248 Recuperado de https://issuu.com/daliadiezdetancredi/doc s/revista_investigaci_n_vol.44-101_sep-dic

EDUCACIÓN 3.0 (24/07/2019) Storytelling: el potencial educativo de contar historias Recuperado de https://www.educaciontrespuntocero.com /noticias/storytelling-educacion-historias/

EDUforics (25 abril 2017) Aprendizaje basado en proyectos: un proyecto auténtico y real Recuperado http://www.eduforics.com/es/aprendizajebasado-proyectos/

Espinosa Mosqueda, R., Rodríguez Venegas, R., y Olvera Maldonado. M., (junio 2017): El uso de las TIC, TAC, TEP, para desarrollar competencias empresariales $y$ comunicativas en los estudiantes universitarios, Revista TECSISTECATL, n. 
21.

Recuperado

de

http://www.eumed.net/rev/tecsistecatl/n2 1/tic-tac-tep.html

García López, R., Armenta, J., Lozano Rodríguez, A., Mercado Varela. M., (2020) Investigaciones sobre ambientes educativos mediados por tecnología. 1a Edición

Gil Mateos, J. E. (2020). Una mirada para el posicionamiento de la tecnología educativa en las universidades. REVISTA CIENTÍFICA ECOCIENCIA, 7(4), 1-14. Recuperado de http://revistas.ecotec.edu.ec/index.php/eco ciencia/article/view/348

González, H. y Guzmán B. (2010). Principios Didácticos para la elaboración de un E-Book en salud sexual y reproductiva. Revista de Investigación, 71(34), 141-167. Recuperado de

http://www2.scielo.org.ve/scielo.php?scrip $\mathrm{t}=\mathrm{sci}$ _arttext\&pid=S1010-

29142010000300008\&lng=es\&nrm=iso.

Granda, L., Espinoza, E., y Mayon, S. (2019). Las TICs como herramientas didácticas del proceso de enseñanza-aprendizaje. Conrado, 15(66), 104-110

Guzmán, B. (2013). Evaluación del Curso Tecnología de Información y Comunicación y Educación Ambiental a Través de sus Productos. Trabajo de ascenso. UPEL-IPC

Guzmán, B. (septiembre 2018) TIC y Medios. Conferencia presentada en 27 Encuentro Nacional de Profesores de Francés organizado y promovido por la Asociación Venezolana de Profesores de Francés. Pozo de Rosas, Miranda.

Innovating Pedagogy (Jan 2016) Aprendizaje Crossover Recuperado de

http://innovating-

pedagogy.wikidot.com/aprendizaje-

crossover 17

López, V. E. (2016). Teoría Subyacente en la Promoción y Divulgación de la Educación Ambiental Informal para la Formación del Ciudadano por medio de las Redes Sociales Digitales (tesis doctoral). Instituto Pedagógico de Caracas, Universidad Pedagógica Experimental Libertador, Venezuela
López, E. y Guzmán, B., (mayo agosto, 2020). YouTube y su utilidad en la promoción y divulgación de Educación Ambiental revista de Investigación 100 vol 44 Recuperado: http://revistas.upel.digital/index.php/revin vest/article/view/8957

López, E. y Guzmán, B., (2019). Redes sociales y su utilidad en la educación ambiental promoción y divulgación informal. Horizontes. Revista De Investigación En Ciencias De La Educación, 3(12), 249 - 266 Recuperado: https://doi.org/10.33996/revistahorizonte s.v3i12.85

Machado E. y Rojas, F. (2018). Visión profesional sobre el uso de las TIC en la praxis educativa, desde la perspectiva de los estudiantes de ciencias pedagógicas. PARADIGMA, 39(1), 229-245. Recuperado de

http://revistas.upel.edu.ve/index.php/para digma/article/viewFile/6787/3884

Marqués, P. (1999). La Tecnología Educativa: Conceptualización, Líneas De Investigación. Recuperado de: http:// peremarques.pangea.org/tec.htm

Marqués, P. (2011). Los medios didácticos y los recursos educativos. Recuperado de $\mathrm{http}: / / w w w . p e r e m a r q u e s . n e t /$ medios $2 . h t m$

Martínez-Olvera, W., Esquivel-Gámez I., Martínez Castillo J., (2014). Aula invertida o modelo invertido de aprendizaje: origen, sustento e implicaciones [Documento en línea]. Recuperado: de https://www.academia.edu/11535968/Aul a_InvertidaoModelo_InvertidodeAprendizaj eorigensustentoeimplicaciones

Montoya, G. (1997). Estrategias De Instrucción. Recuperado de http://es.scribd.com/doc/50451813/estrat egias-de-instruccion

Montoya, E. (2013). Estrategias de Instrucción. Diplomado Vicerrectorado de Extensión UPEL.

Moreira, C., Abuzaid, J., Elisondo, R., \& Melgar, M. (2020). Innovaciones Educativas: Perspectivas De Docentes Y Estudiantes De 
La Universidad Nacional De Río Cuarto (Argentina) Y La Universidad Del Atlántico (Colombia). Panorama, 14(26), 33-50. Recuperado doi: http://dx.doi.org/10.15765/pnrm.v14i26.1 480

Raffino, M. E (2019). Concepto de Teoría de Sistemas. [Blog en línea]. Recuperado de https://concepto.de/teoria-de-sistemas/.

Ramírez M., Montoya J., Valenzuela R., González (2017) IINNOVACIÓN EDUCATIVA Investigación, formación, vinculación y visibilidad Impreso en España - Recuperado https://www.sintesis.com/data/indices/97 88491710974.pdf

Rauseo, R (2003) Propuesta de modelo de libro de texto para las asignaturas del área de administración y gerencia de educación física, deporte y recreación. Trabajo no publicado, Universidad pedagógica Experimental Libertador, Instituto Pedagógico Rural El Macaro. Turmero.

Rauseo, R (2004) Uso del perfil reflexivo crítico por el docente de educación física en su labor educativa. Trabajo de grado de maestría no publicado, Universidad Pedagógica Experimental Libertador, Instituto Pedagógico de Caracas, Caracas.

Rauseo, R. (2015) Currículo y su vinculación con la Formación Universitaria en Educación Física. Estudio Comparativo, UPEL - IPC. Tesis Doctoral no publicada, Universidad Pedagógica Experimental Libertador, Instituto Pedagógico de caracas, Caracas.

Rodríguez Gómez, J., (2006), Innovación, innovatividad y políticas educativas Laurus, vol. 12, núm. 22, pp. 301-315 Universidad Pedagógica Experimental Libertador Caracas, Venezuela

Saraiba, A., (07/12/2019) Cinco claves para hablar de política con niños y adolescentes
CECODAP opinión Recuperado https://cecodap.org/cinco-claves-parahablar-de-politica-con-ninos-yadolescentes/

Sosa H., P. (1974) Plan Keller: sistema de instrucción personalizada en evaluación. Gaceta de Pedagogía, (24), 41-54.

Suárez, J., (2011) Saberes profesionales inmersos en la práctica docente de los profesores de la Universidad Pedagógica Experimental Libertador (tesis doctoral). Instituto Pedagógico de Caracas, Universidad Pedagógica Experimental Libertador, Venezuela.

Stojanovic C., L. (2009) Tecnologías de comunicación e información en educación: Referentes para análisis de entornos virtuales de enseñanza y aprendizaje. Revista de Investigación, 33 (68) Septiembre - Diciembre. Instituto Pedagógico de Caracas. Caracas: UPEL - IPC.

Szczurek, M. (1989). La estrategia instruccional. Investigación y Postgrado, 4. (2). 7-26.

Szczurek, M. (1990, febrero). Tendencias actuales de la Tecnología educativa. Ponencia presentada en las Cuartas Jornadas de Tecnología educativa en Venezuela. Caracas. UCV.

Tovar Palencia, W. (2021). La Radio Educativa en Venezuela y América Latina. REVISTA DE INVESTIGACIÓN, 45(102), 378-381. Recuperado de http://revistas.upel.digital/index.php/re vinvest/article/view/9027/5558

UNESCO (2016, Julio 19). [Página Web en línea]. Disponible:

http://www.unesco.org/new/es/unesco/th emes/icts/teacher-education/unesco-ictcompetency-framework-for-teachers/ 